\title{
DO VEHICLES SENSE PAVEMENT SURFACE ANOMALIES?
}

\author{
Charalambos Kyriakou, Symeon E. Christodoulou, and Loukas Dimitriou \\ University of Cyprus, Nicosia, Cyprus
}

\begin{abstract}
Nowadays, pavement monitoring agencies typically assess pavement quality approximately only once per year. The main reason for this low frequency of inspections is the fact that current methods are expensive and laborious. The paper presents a data-driven framework and related field studies on the use of pattern recognition techniques and smartphone sensor technologies for the detection, classification and georeferencing of roadway pavement surface anomalies. The proposed system provides continuous and reliable information about the five most common roadway pavement surface anomalies which are valuable for pavement management systems and public safety.
\end{abstract}

\section{Introduction}

One of the most significant indicators for road quality is the pavement surface condition, which is determined by the anomalies in the pavement surface that influence the ride quality of a vehicle. Pavement surface anomalies can damage vehicles, cause unpleasant driving and sometimes be the cause for traffic accidents, injuries and/or fatalities.
Further, the condition of pavement surfaces is not timeinvariant, as pavements deteriorate in time from causes linked to location, materials used, traffic, weather, etc. Identifying road anomalies related to pavement surface cracks, rutting, raveling, patching and potholes can improve road condition quality. Distributed mobile sensing with connected vehicles and smartphones could provide a viable solution to the problem at a much lower cost compare to current methods which are expensive and laborious.

As a result of the fast and intense development and usage of smartphones in current years, smartphone technology has gained noteworthy consideration within the transportation, infrastructure, and automotive industries. Smartphones can be utilized in the collection of smartphone and vehicle sensor data, since they come with a range of built-in sensors such as accelerometer, gyroscope and GPS sensors. Further, OBD Bluetooth devices coupled with specialized smartphone applications enable the real-time monitoring of, among others, GPS latitude and longitude, forward and lateral acceleration, vehicle roll and vehicle pitch. This paper investigates the use of smartphone applications, in the interest of

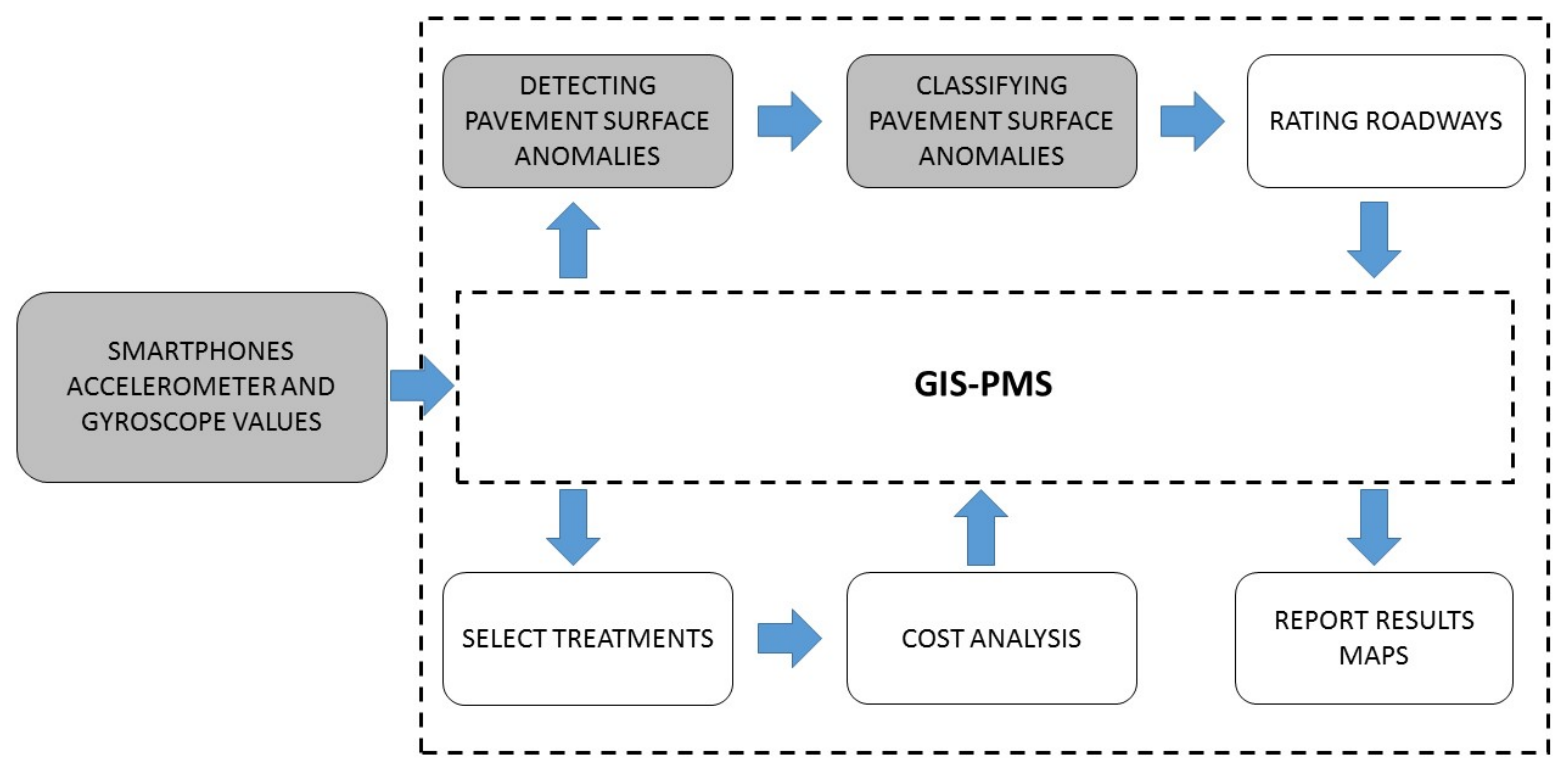

Figure 1: System Components 
improving the condition evaluation and management of roadway pavement surfaces, and by extension it examines the possibility that smartphone-based data and tools may contribute to efficiently monitoring the condition of transport infrastructure.

The goal of roadway anomaly detection by utilization of smartphone technology is set in parallel with the hypothesis that such technology can be used for crowdsourced data collection and analysis in GIS-based pavement management systems (PMS). A general depiction of a PMS is shown in Figure 1, with the focus of this paper highlighted in shaded shapes. As aforementioned, this study focuses on the data collection from smartphones and OBD-II devices for the detection and classification of roadway pavement anomalies. An adequate number of vehicles collecting this crowd-sourced data can then be used to generate a georeferenced event at points where vehicles encounter pavement surfaces anomalies on a roadway network. Even though multiple vehicles may probably provide conflicting data concerning pavement surface conditions, the joint knowledge given by participatory sensing delivers an accurate model of the pavement surface in relation to how an average user experiences the roadway condition.

Further to this introduction to the paper's subject matter, a short literature review section presents an outline of past and ongoing work related to pothole detection using smartphones. A section on methodology describes the developed data collection system and methods, while the results and discussion section includes the processes and tools used to classify the data and experiment outcomes. The paper concludes with key findings and future research directions.

\section{Literature Review}

\section{Roadway pavement defect detection - State of knowledge}

The American Association of Highway Transportation Officials (AASHTO 1993) states that the "...function of a PMS is to improve the efficiency of decision making, expand its scope, provide feedback on the consequences of decisions, facilitate the coordination of activities within the agency, and ensure the consistency of decisions made at different management levels within the same organization." The investment in a PMS is worthwhile as it provides the tools an agency needs in rational resource allocation, optimal use of funds, pavement rehabilitation cost reductions, pavement treatment selection, pavement life extensions, and increased credibility with stakeholders (Washington Department of Transportation 1994). It is essential to understand the benefits and related cost of any expenses in pavement management before starting the process (Khattak, Baladi et al. 2008). In developed countries, PMS are specialised structures supplied with expensive equipment installed on specialised Pavement Evaluation Vehicles (Seraj, van der Zwaag, Berend Jan et al. 2014). The costs linked to a PMS include software (purchase and installation), data collection, database building and system maintenance, as well as, updates, consultant services, employee training, personnel time and actual expenditures on the pavement and rehabilitation (AASHTO 1990).

\section{State of knowledge in automated roadway anomaly detection}

At present, the methodology used for the collection of pavement distress data utilises surveys which address an evaluation or a detailed measurement of distress (Walker, Entine et al. 2002). The collection of the road surface condition data can be either manual (from a moving vehicle, or by "walking" the pavement), or automated (by the use of vehicles fitted with specialized cameras and sensors). Two of the most prominent broad categories of data collection are (1) vibration-based methods and (2) vision-based methods.

The succeeding sections present past and ongoing research work related to the automated detection of roadway pavement surface anomalies by use of low-cost technologies (such as smartphone-based accelerometers and gyroscopes). A summary of these efforts and their findings is listed below, in thematic areas and in chronological order.

\section{Vision-Based Methods}

With regards to vision-based methods, the most relevant work can be found in the works, amongst others, of Radopoulou et al. (Radopoulou, Brilakis et al. 2016), Radopoulou and Brilakis (Radopoulou, Brilakis 2016), Li, Yuan et al. (Li, Yuan et al. 2016), Hadjidemetriou et al. (Hadjidemetriou, Christodoulou et al. 2016, Hadjidemetriou, Vela et al. 2017) and Christodoulou et al. (Christodoulou and Kyriakou et al. 2019).

In Radopoulou et al. (Radopoulou, Brilakis et al. 2016), video data collected from a car's parking camera was u to detect defects in frames, coupled it with elevation signals collected from accelerometers attached to the car to reconstruct the profile of the road, and classified detected defects according to their type and severity. The researchers reported that the initial identification of frames including defects produced an accuracy of $96 \%$ and approximately $97 \%$ precision, and that further experiments on such frames, aiming at the detection of potholes, patches and three different types of cracks resulted in over $84 \%$ overall accuracy and over $85 \%$ precision.

Further, Radopoulou and Brilakis (Radopoulou, Brilakis 2016) presented an application of the Semantic Texton Forests (STF) algorithm for automatically detecting patches, potholes and three types of cracks in video frames captured by a common parking camera, reporting over $70 \%$ accuracy in all of the tests performed, and over $75 \%$ precision for most of the defects.

A vision-based approach was employed by Li, Yuan et al. (Li, Yuan et al. 2016) who proposed a method to integrate the processing of two-dimensional images and of ground penetrating radar (GPR) data for pothole detection. The images and GPR scans are first preprocessed and a pothole 
detector is designed by investigating the patterns of GPR signals, and then the position and dimension of the detected potholes is estimated from GPR data and mapped to the image to enable a localized shape segmentation. The researcher reported a precision, recall, and accuracy of $94.7 \%, 90 \%$ and $88 \%$, respectively.

Hadjidemetriou et al. (Hadjidemetriou, Christodoulou et al. 2016, Hadjidemetriou, Vela et al. 2017) presented a method based on image processing and Support Vector Machine (SVM) classification. The feature vectors used in the classification consist of an image histogram and two texture descriptors, and the output is a binary image where each image block is classified as "patch" or "no-patch". The performance of their proposed method is rated by a detection accuracy of $81.97 \%$, a precision of $64.21 \%$, and a recall of $91.21 \%$.

Finally, Christodoulou et. al. (Christodoulou, Symeon E., Kyriakou et al. 2019) presented a big-data driven methodology for the detection of roadway anomalies, utilizing smartphone-based data and image signal streams. The methodology uses a vision-based method with entropic texture segmentation filters and SVM classification for the detection of patch defects on roadway pavements. The presented system pre-processes video streams for the identification of video frames of changes in image-entropy values, isolates these frames and performs texture segmentation to identify pixel areas of significant changes in entropy values, and then classifies and quantifies these areas using SVM. The developed SVM is trained and tested by feature vectors generated from the image histogram and two texture descriptors of non-overlapped square blocks, which constitute images that include patch and no-patch areas. The outcome is composed of block-based and image-based classification, as well as of measurements of the patch area.

\section{Vibration-Based Methods}

With regards to vibration-based methods for the assessment of roadway pavements, relevant work can be found in the works, among other, by Chen et al. (Chen, Tan et al. 2016), Jang et al. (Jang, Yang et al. 2016), Sigh et al. (Singh, Bansal et al. 2017) and Allouch et al. (Allouch, Koubâa et al. 2017).

A crowdsourced approach using hardware modules mounted on distributed vehicles was utilized by Chen et al. (Chen, Tan et al. 2016). Low-end accelerometers and GPS devices were used to obtain vibration patterns, locations, and vehicle velocities. Further, a light-weight data mining algorithm was utilized to detect road surface events and transmit potential pothole information to a central server. The researchers reported a case-study deployment of their system on 100 taxis, and pothole detection accuracy of $90 \%$ with nearly zero false alarms.

Jang et al. (Jang, Yang et al. 2016) proposed an automated method to obtain up-to-date information about potholes by using a mobile data collection kit mounted on vehicles. In each mobile data collection kit, a triaxial accelerometer and global positioning system sensor, collects data for the detection of street defects. At a back-end server, a street defect algorithm, which relies on a supervised machine learning technique and a trajectory clustering algorithm, enhance the performance of the proposed monitoring system by integrating data collected from multiple sensorequipped vehicles.

Sigh et al. (Singh, Bansal et al. 2017) presented a smartphone based sensing and crowdsourcing technique to detect the road surface conditions. The in-built sensors of the smartphone like accelerometer and GPS have been used to observe the road conditions. The motivation of their work is to improve classification accuracy of detecting road surface conditions using Dynamic Time Warping (DTW) technique which has the ability to automatically cope with time deformations and different speeds associated with time data. Their technique shows detection accuracy rate of $88.66 \%$ and $88.89 \%$ for potholes and bumps respectively.

Allouch et al. (Allouch, Koubâa et al. 2017) motivation was to create a real-time android application 'Roadsense' that automatically predicts the quality of the road based on a triaxial accelerometer and a gyroscope, show the road location trace on a geographic map using GPS, and save all recorded workout entries. Decision tree classifier was applied on training data to classify road segments and to build their model. Their experimental results show consistent accuracy of $98.6 \%$.

\section{Related work published to date by the paper's authors}

The works of Kyriakou et al. (Kyriakou, Christodoulou et al. 2016) explored the use of data, collected by sensors from smartphones and automobiles' onboard diagnostic (OBD-II) devices while vehicles are in movement, for the detection of pavement transverse defects, longitudinal defects and potholes/manholes. The smartphone-based data collection was complimented with artificial neural networks detecting and classifying identified roadway anomalies. Thirty-one factors were used for the detection (subsequently reduced to nine, without loss of accuracy). The proposed method and system architecture were checked against three types of roadway defects and validated against hundreds of roadways run (relating to several thousands of data points) with above $90 \%$ accuracy rate. Their study's results confirm the value of smartphone sensors in the low-cost (and eventually crowd-sourced) detection of roadway anomalies.

Kyriakou et al. (Kyriakou, Christodoulou et al. 2018) discussed the development of a low-cost pavement assessment method and a geographic information system (GIS)-based decision support system (DSS). Data were collected by means of a smartphone and then analyzed for anomalies by use of artificial neural networks, robust regression, and various classification algorithms. The researchers reported pothole-detection accuracy levels of about $90 \%$.

Kyriakou et al. (Kyriakou, Christodoulou et al. 2017a, Kyriakou, Christodoulou et al. 2017b) presented a study on the use of low-cost technology for data collection, 
detection and georeferencing roadway patches and potholes by use of smartphone's accelerometers and gyroscopes, while vehicles are in movement. The proposed approach, which leads itself to participatorysensing applications, utilises readily available and lowcost sensing technologies found in contemporary smartphones, coupled with robust regression analysis, various algorithms and bagged trees classification model to analyse in near-real time the signal of a vehicle's roll and pitch values as the vehicle traverses roadway pavements. The proposed patch and pothole detection method has been piloted on an urban roadway network showing a detection accuracy rate of over $90 \%$, and it is currently expanded to cover larger datasets and a bigger number of roadway defect types.

\section{Proprietary commercial efforts}

The 2014 Mercedes-Benz S-Class used a Light-Detectionand-Ranging (lidar) scanner to measure pavement roughness as a component of an active suspension system. Recently, the Jaguar Land Rover automaker announced that is examining a new connected vehicle technology which permits a vehicle to point dangerous potholes in the road and then allocate this data in real time with other vehicles and road authorities (O'Donnell, Mc Conomy 2017).

\section{Literature review summary and concluding remarks}

The aforementioned systems show that pavement condition assessment by use of smartphone sensors is technologically feasible.

Among the most noteworthy limitations of existing visionbased methods are the need for noise filtering, shadow removal and background illumination correction. These methods either rely on complete 3D surface reconstruction, which comes along with high equipment and computation costs, or make use of acceleration data, which can only provide preliminary and rough condition surveys. Finally, and most important, existing visionbased methods require large storage space and extensive computation for image processing.

The most important limitation of existing vibration-based methods is that they are constrained by tire-hitting paths and by vibration-inducing pavement anomalies (i.e. they are dependent on how the road surface anomaly is hit by the tire, and on the speed of travel). Despite hardware differences in terms of GPS accuracy and accelerometer sampling rate and noise, pothole detection is possible. However, the detection of softer vibration-inducing pavement anomalies has proven difficult. Finally, a speed dependence removal approach must be utilized, especially for the cases involving sudden stops or sudden changes in motion acceleration.

The undertaken research is vibration-based in order to avoid the limitations of vision-based methods and utilizes a new approach to pavement anomaly detection that uses gyroscope readings (roll and pitch values), which are scientifically proven to govern 3Drigid body dynamics, in order to avoid speed dependence and false indications when there is a sudden motion. The suggested system targets the detection of five roadways anomalies (instead of 2-3 of the existing vibration systems) and the development of a GIS-based pavement monitoring system.

\section{Methodological setup}

The section focusses on the detection of five types of common roadway anomalies in six different sections of local roadways and two highway roadways (of $80 \mathrm{Km}$ total distance, $\sim 10$ data points per geographical location, 3349 data points of cracks, 1401 data points of rutting and ravelling, 1770 data points of patches and 2770 data points of potholes), which examines in tandem, albeit being part of a larger effort on the detection of vibration-inducing pavement anomalies, as documented in Kyriakou et al. (Kyriakou, Christodoulou et al. 2016, Kyriakou, Christodoulou et al. 2018, Kyriakou, Christodoulou et al. 2017b, Christodoulou, S. E., Kyriakou 2018, Christodoulou, Symeon E., Kyriakou et al. 2019).

According to the Federal Highway Administration (FHWA) (Miller, Bellinger 2003) there are longitudinal, transverse, block and alligator cracks. FHWA states that ravelling is a progressive loss of pavement material, rutting is displacement of material creating channels in wheelpaths, patches are original surface repaired with new asphalt material and potholes are holes or loss of pavement material caused by traffic loading, fatigue and inadequate strength (Miller, Bellinger 2003).

Data on these types of pavement surface anomalies were collected in-situ by use of a car equipped with a smartphone (mounted on the car's windshield) with its sensors turned on, and with an OBD-II reader attached to it. The smartphone was also fitted with the DashCommandTM application for recording (and exporting) sensor readings of taken data. Vehicle system data transmitted through the OBD-II reader to the smartphone device and then transferred for either processing or storing, via a digital cellular connection or other means. Further, for visually verifying the existence of a pavement surface anomaly (as detected by the sensor data), the smartphone had also its video camera active for recording the routes travelled.

The data, collected at intervals of 0.1 seconds, included a total of 31 uni-dimensional (e.g. X, Y, Z accelerations, speed, etc.) and two-dimensional indicators (e.g. the smartphone's roll and pitch values). At the back-end server, defect detection algorithms based on robust regression analysis, various algorithms and bagged trees classification model enhances the performance of the proposed monitoring system, by integrating data collected from multiple sensors and deducing knowledge from these participatory sensors.

Mathematically, the proposed method is based on rigidbody dynamics. Any three-dimensional rotation can be described as a sequence of yaw, pitch and roll rotations (Figure 2). Pitch is defined as counter clockwise rotation of $\theta_{\text {pitch }}$ about y-axis. Roll is defined as counter clockwise rotation of $\theta_{\text {roll }}$ about x-axis. Yaw is defined as counter 
clockwise rotation of $\theta_{\text {yaw }}$ about z-axis.

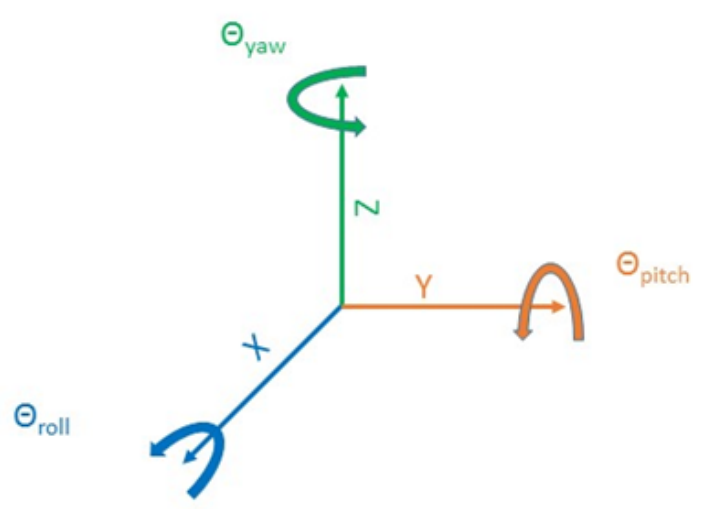

Figure 2: Yaw, pitch and roll rotations (Kyriakou, Christodoulou et al. 2017b)

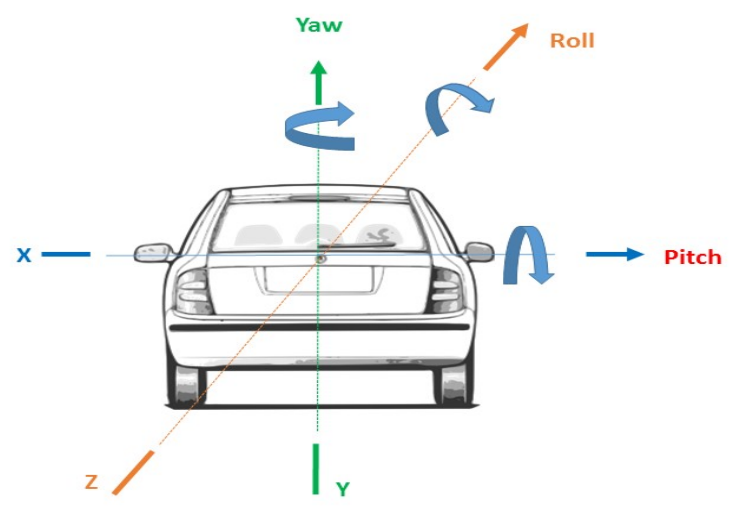

(a)

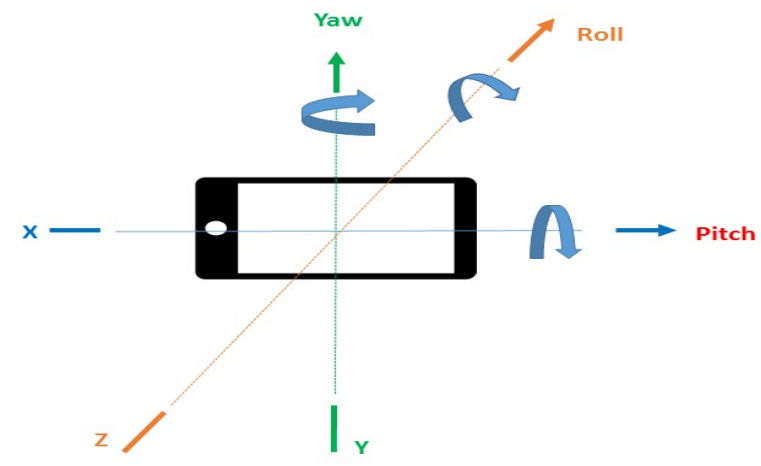

(b)

Figure 3: Smartphone's (a) roll and pitch directions and (b) relation to car's wheels' differential (Kyriakou, Christodoulou et al. 2018)

The smartphone's roll and pitch values (Figure 3a) can be associated with the host car's roll and pitch values (Figure $3 b)$. In essence, the roll metric refers to a car's acceleration variation between its left and right front wheels, while the pitch metric refers to a car's acceleration variation between its front and rear wheels. Concurrently, the roll and pitch values define the way in which the host car is off balance (sideways and front/back).

Robust regression analysis is used to detect the most significant variables and the underlying regression equation. Tukey's Biweight robust influence function was used. It is an M-Estimator that uses iteratively reweighted least squares. This M-estimator completely downweighs observations with large outliers until their weight is set to zero. It provides protection against heavy-tailed error distributions. The regression analysis outputs are shown below (Table 1), listing the most important statistical variables (with $p$-values $\leq 0.05$ ).

Table 1: Most important variables according to robust regression

\begin{tabular}{|l|l|}
\hline Variable & Variable Description \\
\hline VAR_1 & Forward Acceleration (Gs) \\
\hline VAR_2 & Lateral Acceleration (Gs) \\
\hline VAR_3 & Vehicle Pitch $\left(\hat{\mathrm{A}}^{\circ}\right)$ \\
\hline VAR_4 & Vehicle Roll $\left(\hat{\mathrm{A}}^{\circ}\right)$ \\
\hline
\end{tabular}

Robust regression determines the coefficients $\beta \mathrm{i}$ of each factor $x i$, and the residual error $\varepsilon j$ for each observation $j$, in the equation

$\mathrm{y}_{\mathrm{j}}=\beta_{0}+\beta_{1} \mathrm{x}_{1 \mathrm{j}}+\beta_{2} \mathrm{x}_{2 \mathrm{j}}+\ldots+\beta_{\mathrm{p}} \mathrm{x}_{\mathrm{pj}}+\varepsilon_{\mathrm{j}}$,

which is expected to link the dependent variable, $\mathrm{y}_{\mathrm{i}}$, to the independent variables, $\mathrm{x}_{\mathrm{i}}$. The independent variables are the 31 parameters mentioned above, and the dependent variable relates to the classification status of the pavement (' 1 ' for no defect, ' 2 ' for cracks, ' 3 ' for rutting and ravelling, '4' for patching and ' 5 ' for potholes). The subscript ' $\mathrm{j}$ ' denotes the observation (row) number (nearly 3,000 data rows were used in the analysis). Robust regression was selected instead of other regression analysis, because it analyses highly contaminated data by detecting outliers from both dependent and independent variables.

\section{Results and discussion}

The collected raw data exposes the difficulty of the detection problem, as variables such as the lateral acceleration assumed to highlight roadway anomalies are not as definitive as originally thought. A plot of lateral acceleration (Figure 4) helps reveal the absence of any pattern in the taken readings. The vertical variability is random even at a point of known pavement surface anomaly.

Despite the fact the plots indicate areas of suspicion (highs and lows, away from the running average values), they are not reliable indicators. Further, the plots fail to provide information on other running parameters which could affect the accuracy of the data (e.g. the forward and lateral acceleration). 


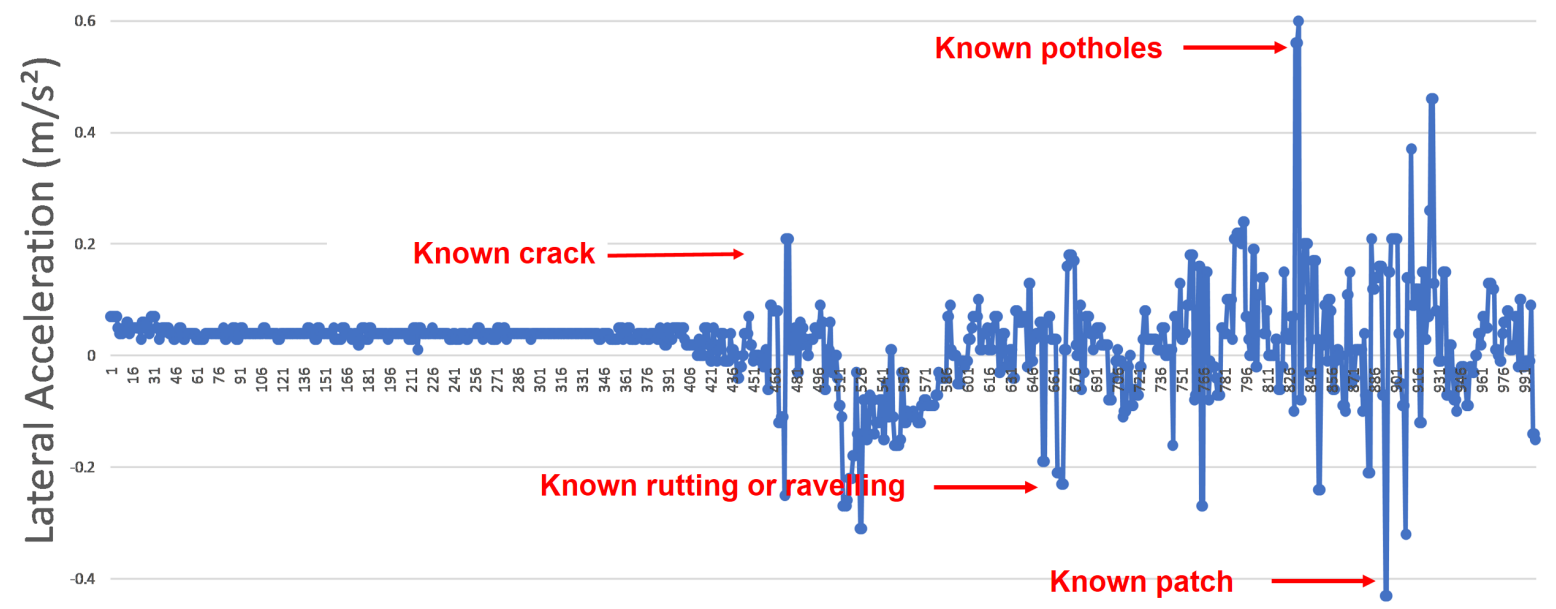

\section{Time $(\mathrm{s})$}

Figure 4: Lateral Acceleration over time

Training/Validating Phase

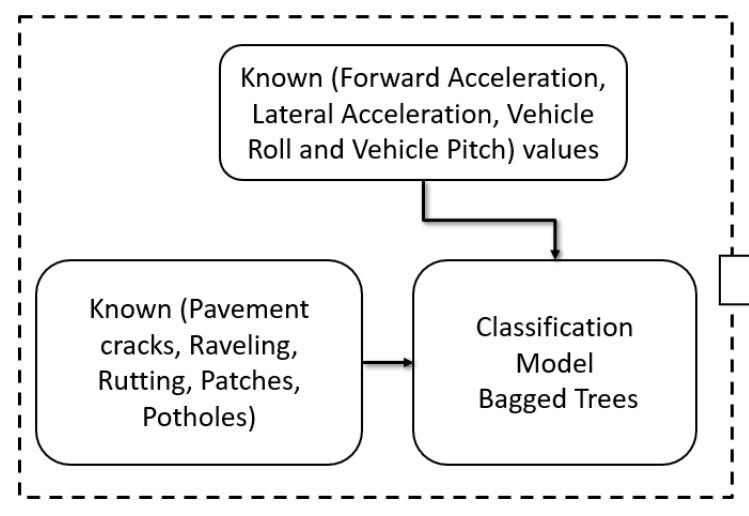

Prediction Phase

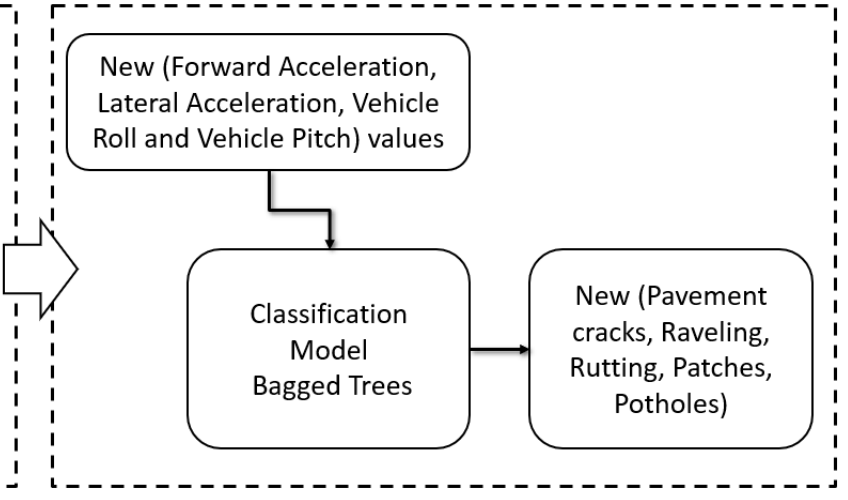

Figure 5: Classification models architecture

\section{Bagged Trees Classification model}

Classification can be achieved with supervised machine learning in which an algorithm "learns" to classify new observations from examples of labeled data. In order to classify and validate the data, supervised learning algorithms for multiclass problems were used and an evaluation comparison was conducted between various classification algorithms to check their performance, including decision trees using various classifiers, discriminant analysis, support vector machines, logistic regression, nearest neighbors, and ensemble classification. Performance was evaluated for both training and validation datasets.

Supervised machine learning was performed by supplying a known set of input data (observations or examples) and known responses to the data (i.e., labels or classes). The abovementioned datasets were fed into classification models consisting of 4 observations and 5 responses. The classification model observations are the parameters listed in Table 1, while the responses are the values of ' 1 ' for no defect, ' 2 ' for cracks, ' 3 ' rutting and ravelling, '4'patching and ' 5 ' potholes. The data used for training the model is the basis for the generation of predictions in response to new data. The classification model architecture (shown in

\begin{tabular}{|c|c|c|c|}
\hline Figure & was & nented & MATLAB $^{\mathrm{TM}}$ \\
\hline Classifier Type & Train Accuracy (\%) & Classifier Type & Train Accuracy (\%) \\
\hline Complex Tree & $99.4 \%$ & Fine KNN & $99.6 \%$ \\
\hline Medium Tree & $99.4 \%$ & Medium KNN & $98.9 \%$ \\
\hline Simple Tree & $91.4 \%$ & Coarse KNN & $88.5 \%$ \\
\hline Linear Discriminant & $79.5 \%$ & Cosine KNN & $98.1 \%$ \\
\hline Quadratic Discriminant & $95.3 \%$ & Cubic KNN & $99.0 \%$ \\
\hline Linear SVM & $87.4 \%$ & Weighted KNN & $99.6 \%$ \\
\hline Quadratic SVM & $99.6 \%$ & Boosted Trees & $71.5 \%$ \\
\hline Cubic SVM & $99.6 \%$ & Bagged Trees & $99.7 \%$ \\
\hline Fine Gaussian SVM & $99.6 \%$ & Subspace Discriminant & $82.5 \%$ \\
\hline Medium Gaussian SVM & $99.6 \%$ & Subspace KNN & $99.6 \%$ \\
\hline Coarse Gaussian SVM & $92.7 \%$ & RUSBoosted Trees & $93.8 \%$ \\
\hline
\end{tabular}

Figure 6: Classifier type training statistics 
After cross-validating the various classification systems and comparing the resulting cross-validation errors of each method, the best model was selected to be the bagged trees classification model (Figure 6). Bagged trees procedure is simple: (1) takes a sample from the dataset, (2) fits the tree to this data set, (3) repeat steps 1 and 2 many times (typically 50-1000), and (4) makes predictions for new data using each of the fitted models and average the predictions. Bagged trees use Breiman's 'random forest' algorithm. 'Random forests are a combination of tree predictors such that each tree depends on the values of a random vector sampled independently and with the same distribution for all trees in the forest. The generalization error for forests converges to a limit as the number of trees in the forest becomes large' (Breiman 2001).

\begin{tabular}{|c|c|c|c|c|c|c|c|c|}
\hline & & $100 \%$ & & & & & $100 \%$ & \\
\hline y & 2 & & $100 \%$ & & & & $100 \%$ & \\
\hline $\bar{d}$ & 3 & & & $100 \%$ & & & $100 \%$ & \\
\hline & 4 & & & & $100 \%$ & & $100 \%$ & \\
\hline & 5 & & & $1 \%$ & & $99 \%$ & $99 \%$ & $1 \%$ \\
\hline & & 1 & 2 & 3 & 4 & 5 & $\begin{array}{c}\text { True } \\
\text { Positive } \\
\text { Rate }\end{array}$ & $\begin{array}{c}\text { False } \\
\text { Positive } \\
\text { Rate }\end{array}$ \\
\hline & & \multicolumn{5}{|c|}{ Predicted } & & \\
\hline
\end{tabular}

Figure 7: Bagged trees confusion training matrix (cracks, rutting and ravelling, patching and potholes)

As shown in Figure 7, the bagged trees classify the three roadway anomalies (target classes ' 2 ', ' 3 ','4' and '5') while also distinguishing the 'no defect' condition (target class ' 1 '), consequently discriminating normal and abnormal roadway pavement surface conditions. For each pavement surface anomaly, data is used for training, for validating and for predicting the bagged trees, with an outline of the obtained classification results presented in Figure 8 .

\begin{tabular}{|l|l|l|l|l|l|}
\hline $\begin{array}{l}\text { Classifier } \\
\text { Type }\end{array}$ & Accuracy & $\begin{array}{l}\text { Total } \\
\text { (data) }\end{array}$ & $\begin{array}{l}\text { True } \\
\text { (data) }\end{array}$ & $\begin{array}{l}\text { False } \\
\text { (data) }\end{array}$ & (\%) \\
\hline $\begin{array}{l}\text { Bagged } \\
\text { Trees }\end{array}$ & Train & 17291 & 17290 & 1 & 99.95 \\
\hline $\begin{array}{l}\text { Bagged } \\
\text { Trees }\end{array}$ & Validation & 17301 & 17300 & 1 & 99.99 \\
\hline $\begin{array}{l}\text { Bagged } \\
\text { Trees }\end{array}$ & Prediction & 11851 & 11832 & 19 & 98.84 \\
\hline & & 46443 & & 21 & \\
\hline
\end{tabular}

Figure 8. Bagged trees training, validation and predictions statistics for the various roadway anomaly cases examined (cracks, rutting and ravelling, patching and potholes)

\section{Spatial DSS and pavement condition-assessment mapping}

Ongoing work involves the development and completion of a spatial decision support system (DSS) for pavement surface condition-assessment mapping. The outcome of the bagged trees analysis and the resulting pavement surface condition assessment score will be mapped spatially, point out the areas of concern and in need of rehabilitation activities (Figure 9).

Further, the generated heatmaps will be used by the pavement agencies to investigate the condition of roadway surfaces and to prioritize their rehabilitation actions according to their budgets.

\section{Conclusions and future work}

This paper focuses on efficiently and cost-effectively appraising the surface condition of roadway pavements and on developing a GIS-based system for roadway anomaly detection and classification. The thesis proposed methodology was designed to provide information and data for analysis, so that highway managers make more consistent and defensible decisions related to the preservation of a pavement network. The suggested

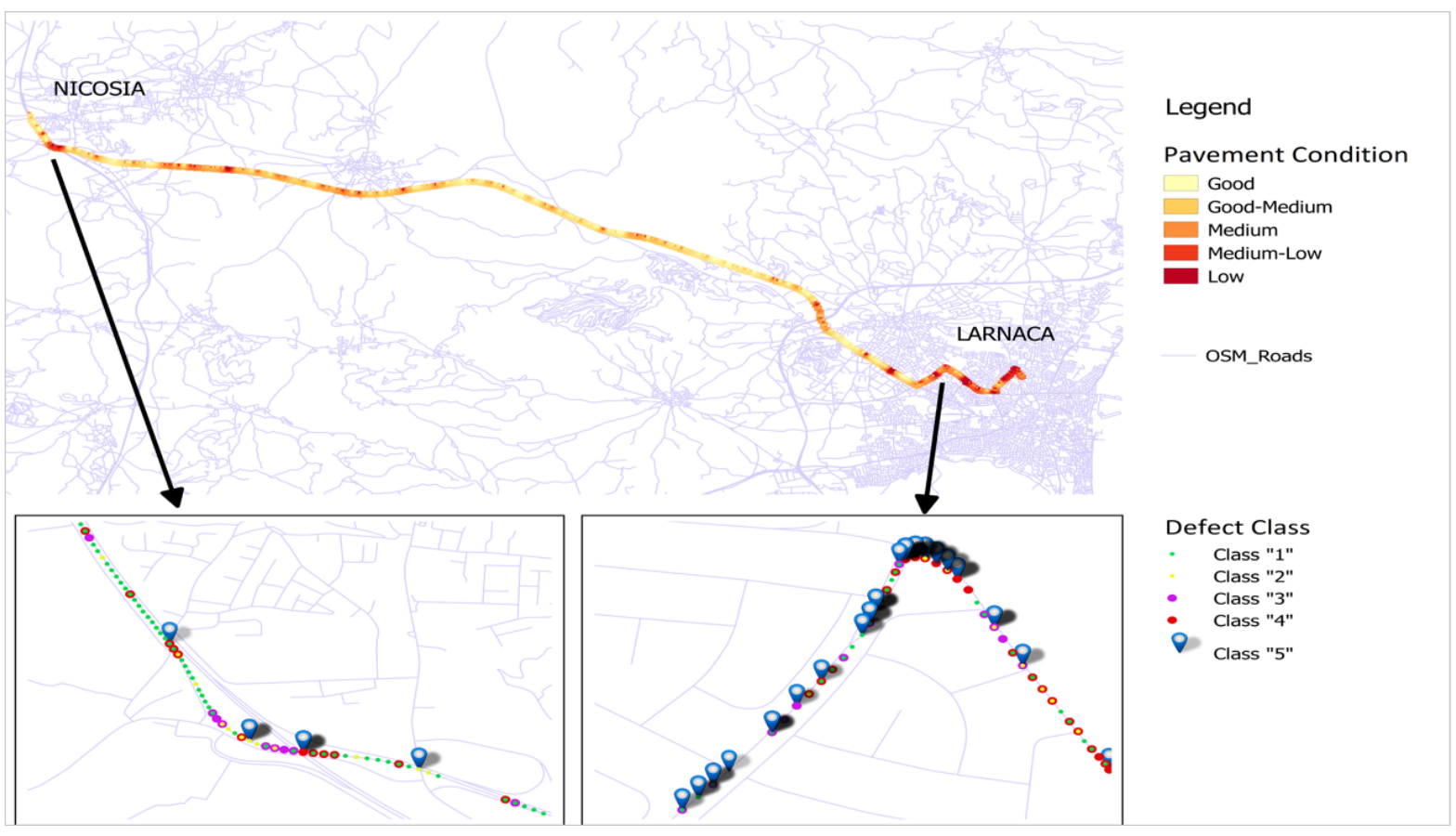

Figure 9. Spatial condition-assessment mapping 
system can collaborate to surveying road condition quality, because is able to identify and classify cracks, rutting, ravelling, patching and potholes on the pavement surface. As a result, this system can raise the value of road surface, protect vehicles from damage and improve traffic safety.

The paper suggested system gives the opportunity to agencies to monitor their roadways networks without buying specialized expensive vehicles which cost over one million dollars, extra software or hardware acquisition and consulting services. The proposed system collected automated pavement surface condition data by using typical passenger vehicles and typical smartphones. Contemporary and future primary datasets of the thesis low-cost pavement monitoring system will be different in form from traditional pavement condition data. Even though connected vehicle data is not likely to directly provide us with traditional assessment metrics (such as IRI and PCI), new metrics might supplement and eventually supplant traditional metrics. An application will be developed in order to collect the specific metrics from the probe-vehicles.

The most important advantage of this system is that pavement surface condition data can be collected and analyzed on a daily base so that suitable treatments can be timely applied. Future work will include also the development of suitable treatments for each roadway anomaly and cost analysis of the corresponding maintenance works. Futher, trigger values will be applied for distinguishing different types of cracks, rutting, ravelling, patches and potholes. Discrete ranges could be converted into a numerical (1-5, 1-10, etc.) scale.

The applied methodology is instantly available, low-cost and precise, and can be utilized in crowd-sourced applications leading to roadway assessment and pavement management systems. The presented study documents the detection of common pavement surface anomalies, exhibiting accuracy levels higher than $90 \%$. The proposed methodology is currently field-tested with larger datasets and a higher number of roadway defect types, with links created to GIS mapping and database management systems for the use of the proposed methodology in PMS.

\section{References}

AASHTO, (1993) Guide for Design of Pavements Structures. Washington, D.C: American Association of State Highway and Transportation Officials.

AASHTO, (1990) Guidelines for Pavement Management Systems. Washington, D.C: American Association of State Highway and Transportation Officials.

Allouch, A., Koubâa, A., Abbes, T. and Ammar, A., (2017) Roadsense: Smartphone application to estimate road conditions using accelerometer and gyroscope. IEEE Sensors Journal, 17(13), pp. 4231-4238.
Chen, K., Tan, G., Lu, M. and Wu, J., (2016) CRSM: a practical crowdsourcing-based road surface monitoring system. Wireless Networks, 22(3), pp. 765779.

Christodoulou, S.E. and Kyriakou, C., (2018) Roadway pothole detection by use of smartphone sensor timeseries data and change-point detection. Accepted for Publication, Journal of Advanced Engineering Informatics.

Christodoulou, S.E., Kyriakou, C. and Hadjidemetriou, G., (2019) Pavement Patch Defects Detection and Classification Using Smartphones, Vibration Signals and Video Images. Mobility Patterns, Big Data and Transport Analytics. Elsevier, pp. 365-380.

Hadjidemetriou, G.M., Christodoulou, S.E. and Vela, P.A., (2016) Automated detection of pavement patches utilizing support vector machine classification. In: Electrotechnical Conference (MELECON), 2016 18th Mediterranean 2016, IEEE, pp. 1-5.

Hadjidemetriou, G.M., Vela, P.A. and Christodoulou, S.E., (2017) Automated pavement patch detection and quantification using support vector machines. Journal of Computing in Civil Engineering, 32(1), pp. 04017073.

Jang, J., Yang, Y., Smyth, A., Cavalcanti, D. and Kumar, R., (2016) Framework of Data Acquisition and Integration for the Detection of Pavement Distress via Multiple Vehicles. Journal of Computing in Civil Engineering, pp. 04016052.

Khattak, J., Baladi, Y., Zhang, Z. and Ismail, S., (2008) A Review of the Pavement Management System of the State of Louisiana - Phase I. Journal of the Transportation Research Board, (2084), pp. 18-27.

Kyriakou, C., Christodoulou, S.E. and Dimitriou, L., (2018) Smartphone-based pothole detection utilizing artificial neural networks. Journal of Infrastructure Systems, 25(3), 04019019.

Kyriakou, C., Christodoulou, S.E. and Dimitriou, L., (2017a) Detecting and Classifying Roadway Pavement Anomalies Utilizing Smartphones, On-Board Diagnostic Devices and Classification Models. In: TRB 96th Annual Meeting Compendium of Papers, 2017-18 to 2017-1-12 2017a, Transportation Research Board 96th Annual Meeting; pp. 14p.

Kyriakou, C., Christodoulou, S.E. and Dimitriou, L., (2017b) Detecting Pavement Patches Utilizing Smartphones Technology and Vehicles. In: Volume I $Ð$ Proceedings of the Joint Conference on Computing in Construction (JC3), July 4-7 2017b, Lean and 
Computing in Construction Congress (LC3), pp. 859866.

Kyriakou, C., Christodoulou, S.E. and Dimitriou, L., (2016) Road Anomaly Detection and Classification Using Smartphones and Artificial Neural Networks. In: TRB 95th Annual Meeting Compendium of Papers, 2016-1-10 to 2016-1-14 2016, Transportation Research Board 95th Annual Meeting, pp. 11p.

Li, S., Yuan, C., Liu, D. and Cai, H., (2016) Integrated processing of image and GPR data for automated pothole detection. Journal of Computing in Civil Engineering, 30(6), pp. 04016015.

Miller, J.S. and Bellinger, W.Y., (2003) Distress identification manual for the long-term pavement performance program. United States. Federal Highway Administration. Office of Infrastructure Research and Development, 2003.

O'Donnell, N. and Mc Conomy, K., (2017) last update, Jaquar Land Rover Announces Technology Research Project to Detect, Predict and Share Data on Potholes Available:

https://www.landrover.com/experiences/news/pothole -detection.html [July/17, 2017].

Radopoulou, S.C. and Brilakis, I., (2016) Automated detection of multiple pavement defects. Journal of Computing in Civil Engineering, 31(2), pp. 04016057.

Radopoulou, S.C., Brilakis, I., Doycheva, K. and Koch, C., (2016) A Framework for Automated Pavement Condition Monitoring. In: Construction Research Congress 2016, pp. 770-779.

Seraj, F., Van Der Zwaag, Berend Jan, Dilo, A., Luarasi, T. and Havinga, P., (2014) RoADS: A road pavement monitoring system for anomaly detection using smart phones. In: International Workshop on Modeling Social Media 2014, Springer, pp. 128-146.

Singh, G., Bansal, D., Sofat, S. and Aggarwal, N., (2017) Smart patrolling: An efficient road surface monitoring using smartphone sensors and crowdsourcing. Pervasive and Mobile Computing, 40, pp. 71-88.

Walker, D., Entine, L. and Kummer, S., (2002) Pavement Surface Evaluation and Rating: PASER manual. Madison WI: University of Wisconsin, Transportation Information Center.

Washington Department of Transportation, (1994) A Guide for Local Agency Pavement Managers, Washington State Department of Transportation, Trans Aid Service Center. The Northwest Technology Transfer Center. 